\title{
Buchbesprechungen
}

\section{Beiträge zur schweizerischen Spitalgeschichte}

Kein Gebiet schweizerischer Medizingeschichte ist in den letzten Jahren so fleißig bearbeitet worden, über keines sind so umfangreiche und gut ausgestattete Monographien entstanden wie gerade über die Spitalgeschichte. Der Anstoß dazu kam von außen her. Wie um 1840 ein neuer Spitaltyp, das Krankenhaus für heilbare Patienten, sich durchzusetzen begann und zu neuen Spitalbauten führte, so verlangte auch die neue Zeit wieder in den meisten Kantonen neue Krankenanstalten, da die alten den technischen Anforderungen nicht mehr angepaßt werden konnten. Der Bau vieler neuer Spitäler in den verschiedensten Gegenden unseres Landes wurde der äußere Anlaß zur historischen Besinnung über den zurückgelegten Weg der Spitalentwicklung. Die meisten dieser Spitalgeschichten entstanden in öffentlichem Auftrag. Solche Darstellungen liegen vor von Solothurn (1930), von den Bürgerspitälern St. Gallen (1945), Bern (1945) und Basel (1946), den Kantonsspitälern Münsterlingen (1936), Zürich (1951), St.Gallen (1953), neuerdings von Schaffhausen (1954) und dem Berner Inselspital (1954). Als besonders bedeutungsvoll erweisen sich eingehende rechtsgeschichtliche Untersuchungen über verschiedene Spitalinstitutionen, wie sie jetzt vor allem für den Zürcher Spital bis 1798 (1952) und den Berner Inselspital (1954) vorliegen. Beim Studium der einzelnen Darstellungen ergibt sich der Eindruck einer merkwürdigen Einheitlichkeit der Entwicklung im gesamten bei großer Mannigfaltigkeit im besondern. Wäre die Kloster- und Ordensspitalgeschichte nur annähernd so gut bekannt wie die Geschichte des bürgerlichen Spitals, könnte heute bereits an eine umfassende schweizerische Spitalgeschichte gedacht werden. Es ist vor allem die hochmittelalterliche Geschichte der Spitalinstitution, die noch eingehenderer Behandlung bedarf, in ihren Motiven wie in ihren verschiedenen Realisationen.

600 Jahre Inselspital Bern. Verfaßt im Auftrag der Inselkorporation von Prof.

Dr. H. Rennefahrt und Prof. Dr. E. Hintzsche. 544 Seiten mit über $80 \mathrm{Ab}$ bildungen. Verlag H. Huber, Bern und Stuttgart 1954. Leinen Fr. 25.-.

Die medizinische Geschichte dieses Spitals schildert Hintzsche unter dem Titel «600 Jahre Krankenpflege im Berner Inselspital», den Stoff in neun Kapitel gliedernd:

1. Das bernische Sanitätswesen im ausgehenden Mittelalter. 2. Die Neuordnung des Spitalwesens in Bern zur Zeit der Reformation. 3. Verbesserung der bernischen Gesundheitspflege zwischen 1560 und 1640. 4. Die Entwicklung des Inselspitals zwischen 1640 und 1709. 5. Das Inselspital im 18. Jahrhundert. 6. Das Inselspital von 1798 bis 1842. 7. Das Inselspital von 1843 bis 1883. 8. Das Inselspital von 1884 bis 1922. 9. Das Inselspital von 1923 bis zur Gegenwart. 
Der Verfasser versucht mit Erfolg, die Geschichte dieses alten Berner Spitals in die Medizin- und Medizinalgeschichte dieses langen Zeitraums hineinzustellen, da zu verschiedenen Zeiten ja ganz verschiedene Ansprüche an eine Spitalinstitution gestellt worden sind. Als private Stiftung einer Frau entstanden, zeigte dieses Spital bis zur Reformation keine irgendwie auffallende, besondere Entwicklung. Auch es machte, vor allem aus finanziellen Gründen, den Weg von der Armenkrankenanstalt zum Pfrundhaus, wie so viele ähnliche Anstalten. In der Reformationszeit wurde es, wie manch anderes auch, durch sequestrierten Klosterbesitz stark vergrößert und wurde nun, vielleicht eindeutiger als irgendein anderes Spital der Schweiz, wieder zur Krankenanstalt bestimmt. Von Anfang an unter die Oberhoheit des Rats gestellt, wurde dieses Spital doch nie eine Art Bürgerspital, sondern blieb in seiner Aufnahmepraxis bemerkenswert weitherzig, auch Landfremden offenstehend, ohne aber deshalb im mittelalterlichen Sinn zum Armen- und Fremdenspital zu werden. Die zweite Merkwürdigkeit dieses Krankenhauses bestand darin, daß es nicht einem einzigen beamteten Spitalarzt unterstellt wurde, sondern daß zur Behandlung verschiedene Ärzte aus der Stadt zugezogen wurden, die schließlich das Inselkollegium bildeten, eine nicht nur für dieses Spital, sondern für das gesamte bernische Medizinalwesen und den medizinischen Unterricht besonders bedeutungsvolle Korporation. Auffallend früh wurde in diesem Spital Anatomie getrieben, mit wechselndem Erfolg auch immer wieder versucht, eine Bildungsstätte für Wund- bzw. Landärzte zu sein. Die Stellung dieses Inselspitals hat bis ins beginnende 19. Jahrhundert unter den übrigen Schweizer Spitälern tatsächlich etwas Besonderes an sich. Wenigen Lesern mag es bei der Lektüre dieser Arbeit zum Bewußtsein kommen, wie mühsam die Aufgabe ihres Autors war, durch welchen Berg von Aktenstücken er sich durcharbeiten mußte, wie schwierig die Gliederung des Stoffes war.

Von besonderm Interesse sind die Schwierigkeiten, die sich für eine solche Institution durch die Bildung eines helvetischen Einheitsstaates ergaben mit der Aufgabe einer neuen Formbildung im 19. Jahrhundert, in welcher das Inselspital den Klinikbetrieb der neuen Hochschule mit ihrer medizinischen Fakultät zu übernehmen hatte. Wie für Basel und Zürich verlangte die neue medizinische Situation der vierziger Jahre des letzten Jahrhunderts eine umwälzende Reorganisation des gesamten Spital- und Verwaltungsbetriebs. Wie diese Aufgabe je nach den örtlichen Gegebenheiten verschieden gelöst wurde, ist von ganz besonderm Reiz. Die Schilderung der verschiedenen Instituts- und Klinikdirektoren lockert den an sich oft etwas spröden Stoff in angenehmer Weise auf. Den Abschluß der Arbeit bildet die Darstellung der neuesten Entwicklung, die wie anderwärts auch in Bern nach neuen Formen des Spitalbetriebs rief und zu einem großartigen Ausbau des Inselspitals geführt hat, dem es nun sechshundert Jahre lang beschieden war, den Bedürfnissen der verschiedenen Zeiten zu genügen als eine unter staatlicher Aufsicht stehende selbständige Korporation. 
Das neue Kantonsspital Schaffhausen. Schaffhausen 1954. Herausgegeben anläßlich der Eröffnung am 3. April 1954. 153 Seiten mit zahlreichen Abbildungen. E. RüMBELI: Geschichtliches; H.Schmid : Hundert Jahre Medizin im Krankenhaus Schaffhausen.

Wenn in Basel das Bürgerspital im letzten Jahrhundert die Funktion eines Kantonsspitals und die Aufgabe von Universitätskliniken übernahm, in Zürich im Beginn des letzten Jahrhunderts aus dem Heiliggeist- und bürgerlichen Armenspital, das freilich vorwiegend Landbevölkerung beherbergte, ein Kantonsspital und schließlich Universitätskrankenhaus wurde, so entwickelten sich in Schaffhausen ähnlich wie in St. Gallen die Kantonsspitäler nicht aus den Bürgerspitälern, sondern aus seit langem bestehenden Fremdenspitälern, denen schon aus räumlichen Gründen eine institutionelle Entwicklung versagt geblieben war. Ähnlich wie das Berner Inselspital war auch das Schaffhauser Fremdenspital nach der Reformation zum eigentlichen Krankenhaus bestimmt und unter die ärztliche Betreuung der Scharfrichter unter stadtärztlicher Oberaufsicht gestellt worden. Wie in Zürich und Winterthur versah auch in Schaffhausen vornehmlich die Familie Volmar das Scharfrichteramt, von der auch die ärztliche Kunst generationenlang ausgeübt wurde. Im 17. Jahrhundert hatte J. J. WEPFER die behördliche Erlaubnis erhalten, im Seelhaus Sektionen an dort verstorbenen Patienten auszuführen, der eigentliche Begründer der weltberühmten Schaffhauser Ärzteschule. Das kleine, erst 1947 abgerissene Gebäude mit seinen fünf bis sechs Kammern, in denen achtzehn bis zwanzig dürftige männliche Kranke aufgenommen werden konnten, war aber wie gesagt keiner Entwicklung fähig. In einem mittelalterlichen Beginenhaus war eine entsprechende Anstalt für Frauen, meist Dienstmädchen, eröffnet worden, die indessen nur etwa sieben Insassen aufnehmen konnte. Die ansehnlichen Mittel dieser Anstalten soll der Rat zeitweise andern Zwecken zugeführt haben. Das Seelhaus freilich war durch eine Institution ausgezeichnet, die in der Schweiz wohl einzigartig war. Die Gesellen fast sämtlicher Handwerker waren in Schaffhausen in sogenannten Bruderschaftskassen vereinigt, die seit dem 16. Jahrhundert mit dem Seelhaus durch Einkaufssummen und ständige Beiträge die vertragliche Abmachung getroffen hatten, daß ihre Mitglieder im Krankheitsfall dort Aufnahme finden könnten und unengeltlich verpflegt werden sollten bis zur Genesung oder zum Tod, so daß der weit überwiegende Teil der Schaff hauser Arbeiterschaft fast seit der Reformation krankenhausversichert war, mit einem Rechtsanspruch auf Krankenhauspflege in Kranheitsnot, unabhängig davon, ob der einzelne Stadtbürger Schweizer oder Landfremder war. Ähnlich wie in St. Gallen wurde um die Mitte des letzten Jahrhunderts aus diesem Fremdenspital zunächst ein Gemeindekrankenhaus und schließlich 1901 ein Kantonsspital, das jetzt ebenfalls zu einem ganz modernen Krankenhaus ausgebaut worden ist. Die medizinische Entwicklung dieses Hauses ist sehr anschaulich vom gegenwärtigen medizinischen Chefarzt H.Schmid geschildert. 


\section{H. Rennefahrt, Geschichte der Rechtsverhältnisse des Inselspitals der Frau Anna Seiler.}

Diese Darstellung macht den ersten Teil der obenerwähnten historischen Monographie des Berner Inselspitals aus und zeigt, wie heute kaum mehr eine Spitalgeschichte ohne rechtshistorischen Abschnitt geschrieben werden sollte. In sieben Kapiteln schildert der Autor: 1. Spitäler vor der Stiftung der Frau Anna Seiler. 2. Das Seilerspital bis zur Reformation. 3. Das Spital seit der Reformation bis 1715. 4. Von 1715 bis 1798. 5. Von der Revolution bis zum Dotationsausgleich (1841) und Inselreglement (1843). 6. Unter der Kantonsverfassung (1846) bis zur Verlegung des Inselspitals und des Außerkrankenhauses auf die Kreuzmatte. 7. Die Insel seit 1888 bis zur Gegenwart.

Ist die Darstellung des mittelalterlichen Krankenhaus- bzw. Spitalwesens in ihrer engen Anlehnung an eine einschlägige Arbeit von A. Schneider kaum befriedigend, fällt vor allem in der Schilderung der Anfänge des Inselspitals eine allzu lokale Betrachtungsweise auf. Die Tatsache, daß Bern an der Berührungslinie zweier Bistümer liegt, und die besonders gelagerten macht- und verkehrspolitischen Probleme dieser Stadt, die auch ihre Spitalgeschichte beeinflussen mußten, sind leider nicht berücksichtigt. Die Angabe, das Inselspital sei praktisch im Gegensatz zu allen andern mittelalterlichen Spitalinstitutionen - von allem Anfang an rein weltlichen Charakters gewesen, bedürfte einer viel sorgfältigeren Beweisführung; Stiftungsurkunde und Organisation des Spitals genügen nicht für eine solche Behauptung. Die Bemerkung, der Stiftungszweck, dürftige Kranke bis zu ihrer Genesung ins Krankenhaus aufzunehmen, sei für die damalige Zeit in der Schweiz einzig dastehend, ist unzutreffend. Daß es nicht immer gelang, praktisch bereits geheilte Insassen wieder aus Spitälern zu entfernen, steht auf einem andern Blatt. So hatte auch das Zürcher Spital nach einem päpstlichen Schreiben vom Jahr 1279 den Zweck, Infirmi et Pauperes, Menschen, die arm und krank waren, ex diversis partibus confluentes aufzunehmen. Organisatorisch auffallend ist beim Inselspital die starke Stellung des Spitalmeisters und das Zurücktreten der üblichen dreigeteilten Verwaltung von Spitalmeister, Spitalpflege und Rat. Von einer eigentlichen Spitalpflege ist kaum die Rede. Die Umwandlung eines ursprünglich zur Aufnahme von dürftigen Kranken bestimmten Spitals in eine Pfrundanstalt, wie sie das Berner Inselspital besonders im 15. Jahrhundert erlebt hat und wie sie auch andernorts auftrat, hatte vermutlich vor allem wirtschaftliche Gründe.

Besonderes Interesse verdient, wie schon erwähnt, die Entwicklung dieses Spitals seit der Reformation, die auch rechtshistorisch eingehend und mit großer Sachkenntnis geschildert wird. Auch hier ist es besonders reizvoll zu verfolgen, wie allgemeine Entwicklungslinien ihren lokalen, durch konkrete Gegebenheiten bedingten Ausdruck finden. Auf die Tatsache, daß das Berner Inselspital in seiner Entwicklung unter den Schweizer Spitälern vielfach eine besondere Stellung einnimmt, wurde bereits hingewiesen. 
E. Wyder-Leemann, Rechtsgeschichte des alten Spitals in Zürich. 191 Seiten. Dissertationsdruckerei Leemann AG, Zürich 1952.

Man wird nachträglich bedauern, daß diese Arbeit nicht Bestandteil der 1951 herausgekommenen offiziellen Zürcher Spitalgeschichte bildet, die durch die gut dokumentierte Darstellung gewonnen hätte. Sie führt freilich nur bis ins Jahr 1798. Die in der Berner Darstellung so interessanten Probleme, die der helvetische Einheitsstaat aufwarf, sind in der Darstellung dieser Zürcher Spitalgeschichte aber noch dargestellt. Die Autorin unterteilt ihren Stoff in sechs Abschnitte: 1. Das Spitalrecht im Mittelalter, wobei das mittelalterliche Spitalrecht dem modernen Recht gegenübergestellt und die Stellung des Spitalrechts im System des mittelalterlichen Rechts untersucht wird. 2. Das Zürcher Spital in seiner ersten Entwicklungsphase - seine Verfassung, Verwaltung und Organisation, seine kirchlichen Verhältnisse und seine Spitalinsassen. 3. Das Zürcher Spital vom 13. Jahrhundert bis zur Reformation - Verfassung, Verwaltung und Organisation, kirchliche Verhältnisse am Spital, ärztliche Fürsorge, Recht der Spitalinsassen und Ökonomie des Spitals. 4. Das Zürcher Spital von der Reformation bis zu seiner Neuorganisation im Jahr 1785 - Verfassung, Verwaltung und Organisation, kirchliche Verhältnisse, ärztliche Betreuung, Recht der Insassen, Spitalökonomie. 5. Die Neuorganisation des Spitals zu Ende des 18. Jahrhunderts. 6. Schlußbetrachtungen. - Am Anfang ihrer Arbeit gibt die Autorin einen historischen Überblick über die äußere Entwicklung des Zürcher Spitals, während in einem Anhang Dokumente zur Spitalgeschichte publiziert werden über die älteste urkundliche Erwähnung des Spitals, die Pfründnerordnung von 1528, die Pflegerordnung von 1591, eine Insassenstatistik, Zehnten und Spitalrechnungen von 1500 bis 1750, im Auszug.

Weitere ähnliche Untersuchungen erscheinen sehr wünschenswert. Wie verschieden die Verhältnisse bei den einzelnen Spitälern vor allem im Mittelalter gewesen sein müssen, ergibt etwa ein Vergleich zwischen dem Zürcher und dem Solothurner Spital. Eine weniger rein lokale und vermehrt vergleichende Betrachtungsweise mit besserer Berücksichtigung der vorliegenden Literatur dürfte am ehesten Fortschritte in unserer Erkenntnis erwarten lassen. Dabei ist aber die saubere Methode der Autorin dieser Rechtsgeschichte des Zürcher Spitals besonders lobend hervorzuheben. In ihrer Schlußbetrachtung drückt sie die Ansicht aus, das Zürcher Spital habe seine kirchliche Struktur bis gegen Ende des 14. Jahrhunderts bewahrt, sei aber schon im 15. Jahrhundert völlig weltlichen Charakters geworden, obgleich ihm die Peterskirche inkorporiert gewesen sei. Eine eingehendere kirchenrechtliche Fundierung dieser Ansicht wäre erwünscht. Abzuklären wäre vor allem die Frage, inwiefern im ausgehenden Mittelalter eine äußerlich verweltlichte Institution in kirchenrechtlichem Sinn noch immer geistlich-kirchlichen Charakter haben konnte. Die Autorin betont die völlige Unabhängigkeit des Zürcher Spitals von jedem kirchlichen Einfluß. Auch hier ist die 
Beweisführung nicht zwingend, wenigsten für die Anfänge des Spitals nicht. Konnte auch eine bischöfliche Untersuchungskommission im Jahr 1291 eine Abhängigkeit des Spitals von der Großmünsterpropstei nicht feststellen, so verdient allein schon die Tatsache Beachtung, daß eine solche Untersuchung überhaupt nötig war. Der älteste Spital in Zürich versah offenbar die Funktionen eines Stiftsspitals, hat doch der Propst mit zwei Chorherren regelmäßig darin Brotverteilungen vorgenommen. Ob 1291 die Spitalbruderschaft noch bestand, ist ungewiß. Das Gutachten der Kommission besagt sehr vorsichtig, daß damals eine Bindung des Spitals an das Großmünster nicht nachweisbar war, nicht aber, daß sie nie bestanden hat. Die vorhandenen Quellen gestatten es nicht, einen solchen Zusammenhang zu belegen; nicht gesicherter ist aber die Behauptung des Gegenteils. Man wird gut tun, diese Frage offen zu lassen.

Von Interesse wäre auch eine nähere Würdigung des Gnadenbriefes König Wenzels von Böhmen für das Zürcher Spital aus dem Jahr 1384 gewesen. Dieser Brief ist nicht nur als Ausdruck besonderer Initiative des Spitals, um immer größern Einfluß auf das weltliche und geistliche Leben Zürichs zu erlangen, aufzufassen, sondern ebensosehr als Ausdruck einer besondern königlichen Spitalpolitik, liegt doch aus derselben Zeit auch eine entsprechende Urkunde für das Wiener Spital vor.

Die Angabe der Autorin, ein Jаков Rüтт sei gleichzeitig Spitalscherer, Stadtschnittarzt am Spital, Archiater und Poliater gewesen sei, entspricht natürlich nicht den Tatsachen. JАков RUEFF, wie er tatsächlich heißt, war weder Archiater noch eigentlicher Poliater, hat aber offenbar im Spital auch die Leibeskranken behandelt. $\mathrm{Da}$ es in Zürich zu jener Zeit an geeigneten Chirurgen gefehlt hätte, um das Amt des Spitalscherers und des Stadtschnittarztes getrennt zu besetzen, ist keineswegs richtig; Zürich war an tüchtigen Chirurgen gerade damals sogar auffallend reich. RuEFF war aber zweifellos ein besonders tüchtiger Mann, gehört er doch nicht nur der universellen Medizingeschichte, sondern auch der schweizerischen Literaturgeschichte an. Er war von 1532 bis zu seinem Tod im Jahr 1558 Stadtschnittarzt und als solcher auch am Spital tätig. Seit 1554, eventuell vorher, hat er vermutlich auch die Leibeskranken behandelt, an Stelle des 1554 zum Stadtarzt erwählten Conrad Gessner, zu dessen Pflichten diese Aufgabe gehört hätte laut Bestallungsbrief, der sie indessen offenbar nie erfüllt hat, kaum gegen den Willen der Behörden, sondern wegen seiner vielfältigen anderweitigen Verpflichtungen. Als Spitalärzte werden für jene Jahre LienHARD Ringli und Felix Stoll erwähnt.

Nochmals sei aber hervorgehoben, daß es sich um eine sehr wertvolle Arbeit handelt. Die kleinen Aussetzungen und Diskussionen sollen nur des Referenten besonderes Interesse an derselben belegen.

Bernhard Milt 